\title{
Nonfatal myocardial infarction in previously healthy adolescent - a lyear follow-up
}

\begin{abstract}
The development of acute myocardial ischemia in a child with structurally normal coronary arteries is rarely described in medical literature. Myocardial infarction in a pediatric patient group with angiographically normal coronary arteries could occur because of hypercoagulable states in nephrotic syndrome and antiphospholipid syndrome. The following case report highlights rare but important causes of myocardial infarction in a previously healthy child with normal coronary arteries - coronary artery spasm, with a good outcome.
\end{abstract}

Keywords: acute myocardial ischemia, outcome, adolescent
Volume 5 Issue I - 2018

\author{
Sergey Ivanov,' Natalya Kukhtinova, ${ }^{2}$ Yury \\ Gorbatykh' \\ 'Cardiac Surgery Department of Congenital Heart Diseases, \\ Russian Federation, Russia \\ ${ }^{2}$ Department of Pediatrics, Novosibirsk State Medical University, \\ Russia
}

\begin{abstract}
Correspondence: Natalya Kukhtinova, Department of Pediatrics, Novosibirsk State Medical University, 630090, Novosibirsk, Krasny Prospekt, Russia, Tel +7 (903) 9374304, Email natalya_kuhtinov@mail.ru
\end{abstract}

Received: December 26, 2017 | Published: January 16, 2018

\begin{abstract}
Abbreviations: AMI, acute myocardial infarction; CDC, centre for disease control; ECG, electrocardiography; CAD, coronary artery disease; LV, left ventricle; CHD, coronary heart disease; ACAOS, anomalous coronary artery originating from the opposite sinus
\end{abstract}

\section{Introduction}

The estimated incidence of myocardial ischemia in the population of children and adolescents in Russian Federation is $4.48 \%{ }^{1}$ According to the Centre for Disease Control and Prevention (CDC), mortality from acute myocardial infarction (AMI) is 0.2 deaths per 100,000 of the population in persons aged 15-24years and fewer than 0.2 deaths per 100,000 in infants younger than 1 year. ${ }^{2}$ The incidence of adolescent AMI was found to be 157 cases peryear, or 6.6 events per 1million patient-years. ${ }^{3} \mathrm{AMI}$ in children is most often the severe adverse event amongst all their serious medical conditions. At the same time, there are very few reports of AMI in healthy adolescents. ${ }^{4}$ Specifically, the treatment of acute AMI in children remains largely opinion-based with little evidence to guide therapy. The aim of this paper is to present a case for large-focal AMI in teenagers with intact cardiac muscle and coronary vessels.

The patient's medical history is very average. This 12 year old boy is the only child of healthy young parents, he was vaccinated according to the national calendar, educated in an ordinary school, did not take part in professional sports, no childhood misfortune or child maltreatment was recorded. The family follows a healthy lifestyle. The boy woke up from sleeping due to intensive chest pain and upper extremity discomfort; he was admitted to the emergency department. Chest X-ray and computed tomography showed no pathological changes in the lung tissue and no traumatic injuries to the ribs or spine. Electrocardiography (ECG) was recorded and interpreted promptly. In leads I, II, AVF, V4-V6 evident ST-segment elevation with reduced voltage QRST, Q wave MI. Myocardial cell injury biomarkers such as cardiac troponin ( $\mathrm{cTn}$ I or T) or the MB fraction of creatinekinase (CKMB) was not available at this medical unit. With the diagnosis of coronary artery disease (CAD), acute Q-positive MI of the side and front left ventricle walls, the patient was urgently transferred to a large tertiary cardiac center for intensive care and further diagnostic procedures.

Dynamic multiple ECGs confirmed the signs of MI: large-focal MI of posterolateral wall (Figure 1). The biochemical analysis showed a general increase in CKMB 128U/L (upper reference limit 24U/L); Troponin-T quantitive test was positive. The parameters of lipid and coagulation profile were normal.

According to echocardiography heart cavity size was normal, no significant areas of violation of local and global left ventricular contractility were revealed. There was detected a slight pericardial leaflets separation beyond the basal posterior wall of the left ventricle (LV) $-0.7 \mathrm{~cm}$. Magnetic resonance imaging confirmed the presence of extensive areas of intramyocardial and subepicardial edema in the apical segments 4, 5, 6, 10,11, 12; intramural pathological zone and subepicardial uptake of contrast zone localized in 4, 5, 6 segments, partially in $11,12,13$ segments on the apex of the $\mathrm{LV}$ with a reduction of its longitudinal contractility: $0.5 \mathrm{~cm}$ (upper reference limit $1.0 \mathrm{~cm}$ ). One-stage ECG-synchronized myocardial perfusion scintigraphy showed multiple small areas of LV hypoperfusion, more markedly in the 5,11 segments of inferolateral wall.

Coronary angiography excluded such possible causes of MI as malformations and aneurysms of the coronary vessels, there was angiectasia both in left and right brains CA (Figure 2). Thus, it excluded myocarditis, abnormal structure and atherosclerotic changes in the coronary vessels, and confirmed the diagnosis of large-focal myocardial infarction 2 type.

After comprehensive treatment: bisoprolol, perindopril, aspirin, clopidogrel, amlodipine, spironolactone, trimetazidine, rapid positive dynamics of the general condition, the ECG parameters and values of bio- chemical markers of myocardial necrosis were obtained. The patient was discharged after 12days in the hospital in a satisfactory condition under the supervision of the local cardiologist. At the 12 month follow up : normal CKMB and cTn value, echocardiography 
had not detected any complications of acute MI (the presence of regional wall motion abnormality, thinning or scars) (Figure 3).

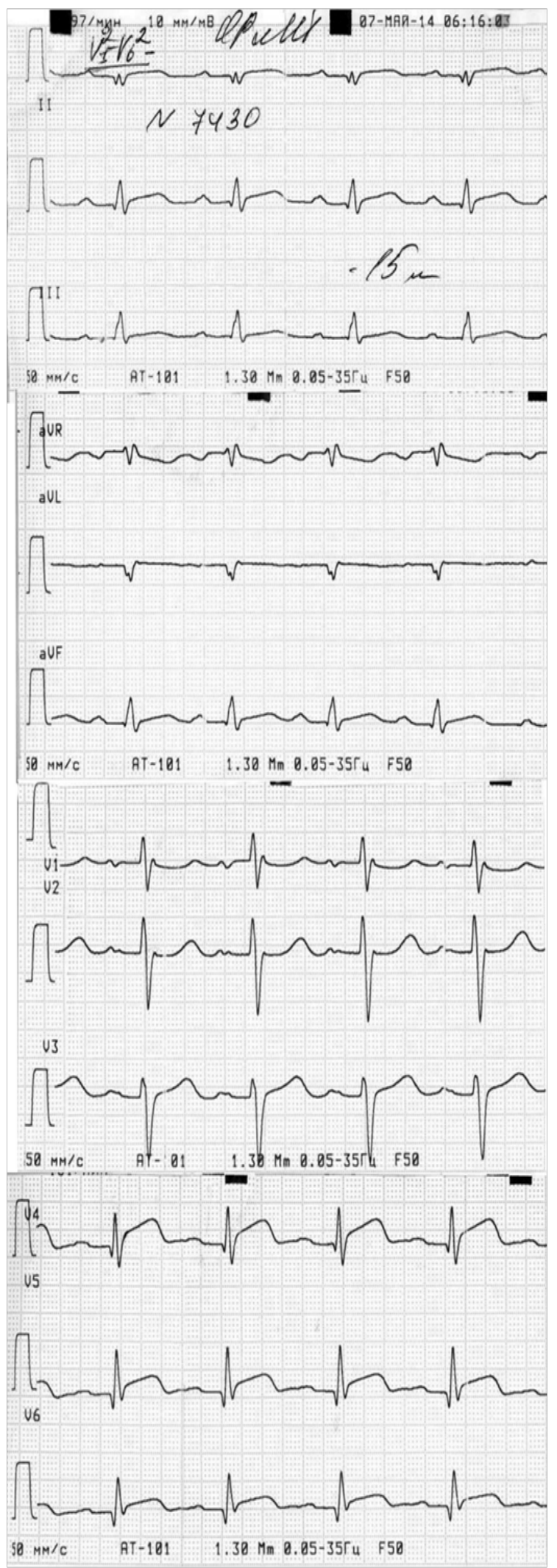

Figure I ECG performed the first day of illness. Marked ST-segment elevation with reduced voltage QRST in leads I, II, AVF,V4 -V6.
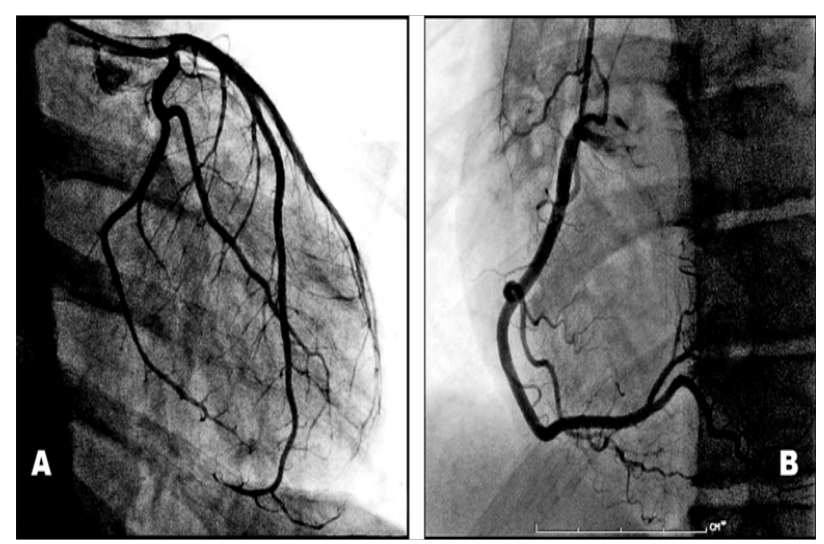

Figure 2 Coronary angiography left (A) and right (B) coronary arteries demonstrated no evidence of significant coronary artery disease.

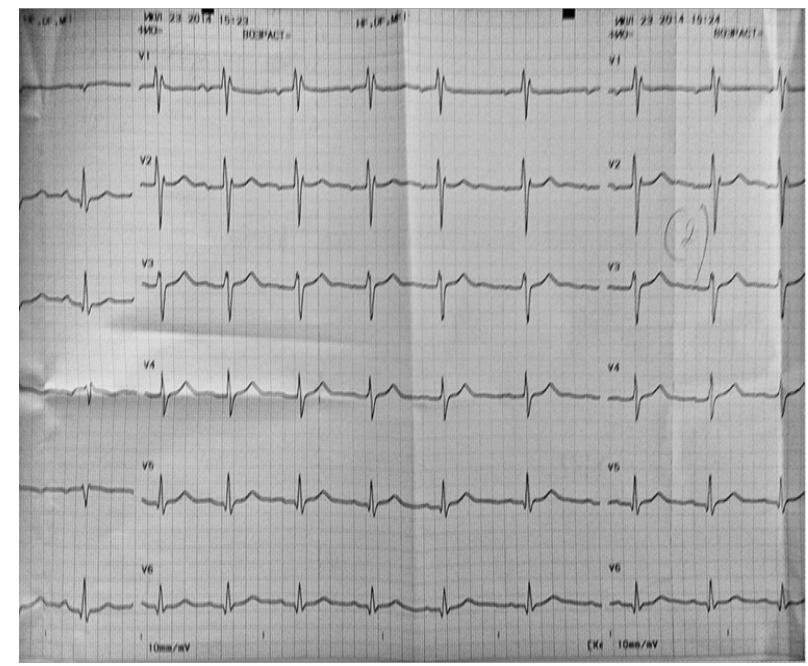

Figure 3 ECG performed I2months after AMI. Normal ECG.

\section{Discussion}

An epidemic of coronary heart disease (CHD) began during the $20^{\text {th }}$ century in most industrialized countries, where CHD is a leading cause of mortality among adults. ${ }^{5}$ AMI in pediatric practice remains a rare disease. Myocardial necrosis is relatively common finding in infants who die due to congenital heart disease, perinatal asphyxia, coronary artery abnormalities, or sepsis. MI prevalence in infants undergoing autopsy is $11 \% .{ }^{6}$ These findings suggest that the course of the MI in newborns and infants may be fatal and can crucially change the prognosis of this major disease. There were reported two cases in which the infant had a normal heart with normal coronary arteries. ${ }^{7} \mathrm{~A}$ review of previously described cases suggests that the most frequent cause of neonatal myocardial infarction is coronary artery occlusion secondary to paradoxical thromboembolization. It is speculated that infarction also can result from coronary hypoperfusion in asphyxiated infants. ${ }^{8}$ David P Riple reported after a long-term follow-up, AMI in the neonate with an anomalous coronary artery originating from the opposite sinus of Valsalva (ACAOS), 24 of those had myocardial infarction with a $4.3 \%$ mortality rate over $4.6 y$ ears. ${ }^{9}$ Krasuski also demonstrated a substantially higher mortality ACAOS rate of $44 \%$ over a mean 9.2year follow-up. ${ }^{10}$

At the same time, there are known a great variety of different conditions that might lead to an MI in other age groups, including Kawasaki disease, ${ }^{11}$ aortoarteritis, myocarditis, 
familial hypercholesterolemia, inherited arteriopathy, primary cardiomyopathy, systemic vasculitis involving the coronary arteries, drug addiction, nephrotic syndrome, storage disease, progeria, sespsis, neoplastic processes. ${ }^{12}$ These diseases were not found in our patient after full clinical, laboratory and instrumental studies. There were no signs of the risk criteria of premature myocardial infarction, such as overweight, high blood pressure or left ventricular hypertrophy. AMI in our patient occurred in the absence of any apparent underlying cause: the etiology of these cases is unclear. As in our case report, the most likely mechanism of myocardial ischemia is considered the intense sympathetic stimulation and transient coronary vasospasm. ${ }^{13}$ All of the above gave a good prognosis.

\section{Conclusion}

The prognosis of AMI in children is crucially dependent on the cause and speed of diagnoses.

\section{Acknowledgements}

None.

\section{Conflict of interest}

Author declares that there is no conflict of interest.

\section{References}

1. Maslennikov GP. The incidence of myocardial ischemia in children and adolescents. Bulletin of the Orenburg State University. Orenburg, Kazakhstan; 2006;13(63):256

2. http://www.disastercenter.com/cdc/aacutcar.html

3. Mahle WT, Campbell RM, Favaloro-Sabatier J. Myocardial infarction in adolescents. $J$ Pediatr. 2007;151(2):150-154.
4. Lane JR, Ben-Shachar G. Myocardial Infarction in Healthy Adolescents. Pediatrics. 2007;120(4):e938-943.

5. Heron M. Deaths: Leading Causes for 2012. National Vital Statistics Reports. 2015;64(10):1-94.

6. Bamber AR, Pryce J, Cook A, et al. Myocardial necrosis and infarction in newborns and infants. Forensic Sci Med Pathol. 2013;9(4):521-527.

7. Kilbride M, Way G, Merenstein G, et al. Myocardial Infarction in the Neonate With Normal Heart and Coronary Arteries. Am J Dis Child. 1980;134(8):759-762.

8. Abbal J, Paranon S, Brierre G, et al. Myocardial Infarction in a newborn from a diabetic mother. Cardiol Young. 2010;20(4):451-454.

9. Ripley DP, Saha A, Teis A, et al. The distribution and prognosis of anomalous coronary arteries identified by cardiovascular magnetic resonance: 15 year experience from two tertiary centres. $J$ Cardiovasc Magn Reson. 2014;16:34.

10. Krasuski RA, Magyar D, Hart S, et al. Long-term outcome and impact of surgery on adults with coronary arteries originating from the opposite coronary cusp. Circulation. 2011;123(2):154-162.

11. Kwak J, Song J, Kang I, et al. Changes in Coronary Perfusion after Occlusion of Coronary Arteries in Kawasaki Disease. Yonsei Med J. 2014;55(2):363-369.

12. Kocabas A, Kardelen F, Akçurin G, et al. Chest pain with myocardial ischemia in a child: should we think about coronary slow flow phenomenon? Türk Kardiyol Dern Ars, - Arch Turk Soc Cardiol. 2013;41(7):646-650.

13. Kristian Thygesen, Joseph S Alpert, Allan S Jaffe, et al. Third universal definition of myocardial infarction. Eur Heart J. 2012;33:2551-2567. 